\title{
Energetic Nanoparticles and Nanomaterials for Future Defense Applications
}

\author{
Marc Comet $^{1}$ (D) Cédric Martin $^{1} \cdot$ Fabien Schnell $^{1} \cdot$ Denis Spitzer $^{1}$
}

Received: 10 December 2018 / Revised: 22 January 2019 / Accepted: 25 January 2019 /Published online: 8 February 2019

(C) The Author(s) 2019

\begin{abstract}
The integration of nanostructured materials in defense systems is expected to improve their performance in terms of power, safety, and reliability. That is why considerable research effort has been undertaken by major military powers worldwide in this domain. The first important step was to develop the production capacities of organic explosives in the state of fine powders with submicron to nanosized particle size distributions. The Spray Flash Evaporation (SFE) process, which is a unique method for producing such materials, was developed at industrial scale. Explosive nanopowders obtained by this process were subsequently mixed with nanosized pyrotechnic compositions such as nanothermites, to prepare hybrid detonating materials able to replace lead-based primary explosives. Composite propellants can also be prepared by SFE which allows mixing their components in a single step with better homogeneity. The ultimate challenge is to move from powder to object, in order to integrate energetic nanomaterials in operational systems. Although the research in this last domain is still in its infancy, several ways of preparation of objects from nanothermites have been recently reported in scientific literature. The focus will be on two examples studied in our laboratory. The first one is the preparation of nanothermites in the state of solid, porous foams; the second one is the use of nanothermites for coating grains of propulsive powder to change their combustion properties.
\end{abstract}

Keywords Nanomaterials $\cdot$ Explosives $\cdot$ Nanothermites $\cdot$ Pyrotechnics

\section{Introduction}

In the domain of energetic materials, the research has mainly focused for one century and a half on the synthesis of new molecules. The most glaring exception to this trend was the invention of dynamite by Alfred Nobel, who stabilized nitroglycerine into different porous nanomaterials and thus became the first scientist to have a materials approach of explosives [1].

The era of nanomaterials has open new horizons to the science of explosives and pyrotechnic compositions and is deeply changing the ways to think and to do in this field. The future energetic substances will be "smart materials" with high performances, high safety and reliability of use, and minimized impact on environment. The first challenge to

Marc Comet

marc.comet@isl.eu

$1 \quad$ NS3E laboratory (UMR 3208 ISL/CNRS/Unistra), French-German research institute of Saint-Louis (ISL), 5 rue du Général Cassagnou, BP 70034, 68301 Saint-Louis Cedex, France overcome was to have particles with size distributions below $1 \mu \mathrm{m}$. For this purpose, a revolutionary process called the Spray Flash Evaporation (SFE) process for nanostructuring explosives and the components of energetic compositions was developed over the last decade [2,3]. The second challenge was to use these elementary bricks to prepare hybrid composite nanomaterials, by mixing the explosive nanopowders with nanothermites. These new detonating compositions, which were called NSTEX (NanoStructured Thermites and Explosives), have unconventional properties in comparison to those of classical explosives and pyrotechnic compositions, which make them promising candidates for replacing primary explosives containing heavy metals such as lead or cobalt [4]. The last challenge is to move from nanopowders to objects, in order to integrate energetic nanomaterials in real systems produced at industrial scale. This specific aspect will be first discussed through the example of the chemical synthesis of nanothermites in the form of combustible foams from aluminum nanopowder and orthophosphoric acid $\left(\mathrm{H}_{3} \mathrm{PO}_{4}\right)[5,6]$. A second example dealing with the coating of a propulsive powder by a nanothermite will be given [7]. 


\section{Preparation of Fine Explosive Powders}

The preparation of high explosives in the state of fine powders $(0.05-1 \mu \mathrm{m})$ is difficult owing to the specific properties of these compounds. First, most of organic explosives are molecules which cannot be polymerized: it is therefore impossible to prepare them by a sol-gel approach, unless using a gelling substance as template $[8,9]$. For this reason, explosive nanopowders prepared by this technique are never chemically pure. The milling technology is hazardous to carry out with explosives for obvious safety reasons. Samples must be prepared by operating in successive small batches. Furthermore, in a milling process, large particles are broken in smaller ones, which are afterward agglomerated in larger aggregates by the pressure exerted by the impact of milling beads. An intrinsic problem of the milling technique is the contamination of samples by the matter coming from different sources: beads, grinding bowl, and liquid used to desensitize the energetic material during the process. The anti-solvent method consists to induce the fast precipitation of an explosive dissolved in a solvent, by gradually pouring the solution into an anti-solvent of the explosive $[10,11]$. This technique generally leads to micron-sized powders, with inclusions of solvent. The spray techniques are the most efficient for producing explosives in fine powders. They differ by the technique used to evaporate the solvent. In spray-drying, the aerosol is dried progressively by a hot gas flow $[12,13]$. The particles formed have spherical shapes and typical size of $1 \mu \mathrm{m}$. The rapid expansion of supercritical solution (RESS process) lead to fine powders with submicron-sized to nanosized particles. The main drawback of this technique is the use of important amounts of supercritical fluid $\left(\mathrm{CO}_{2}\right)$ for preparing limited quantities of sample [14, 15]. None of these techniques can be used for preparing explosive fine powders at industrial scale.

The SFE process is the first technique that allows preparing fine and pure explosive powders, in amounts of typically $100 \mathrm{~g}$ per hour. The underlying principle is to spray in a chamber maintained under dynamic vacuum an aerosol of preheated solvent containing one or several explosives dissolved in it. The ultrafast evaporation of the solvent induces the rapid crystallization of the explosive into small particles which do not have time to grow, and have typical sizes ranging from $50 \mathrm{~nm}$ to less than $1 \mu \mathrm{m}$. The most used solvent for preparing explosive nanopowder by SFE is acetone, whose physicochemical properties are particularly well suited for this technique. To date, trinitrotoluene (TNT), pentaerythritol tetranitrate (PETN), hexogen (RDX), octogen (HMX), hexanitrohexaazaisowurtzitane (CL-20), ammonium dinitramide (ADN) were prepared by SFE (Fig. 1) [2, 16-18].

One of the advantages of SFE process relies in the possibility to produce fine powders with different particle morphology. For instance, core-shell particles of hexolite, in which RDX is the core and TNT is the shell, were prepared in a single step [19]. A co-crystallized structure $(1: 2 \mathrm{~mol} / \mathrm{mol})$ was obtained from HMX and CL-20, which confirmed that molecular recognition occurs in short times [20]. These examples show the outstanding versatility of this process.

Composite LOVA propellants were also prepared by SFE from solutions of nitrocellulose, a plasticizer (2,4-dinitro-2,4diazahexan: DNDA6) and hexogen, in ethyl acetate [21]. In this domain, SFE makes possible the preparation of propulsive powders with a better homogeneity, in a single step.

\section{Use of Fine Explosive Powders for Preparing Hybrid Energetic Nanocomposite Materials}

Fine explosive powders prepared by SFE process were mixed with nanothermites to obtain hybrid energetic nanocomposite materials, which were called NSTEX (NanoStructured Thermites and Explosives). This concept originates from the research of Comet et al., who have proposed to solidify hexogen in a porous matrix of chromium (III) oxide firstly synthesized by the combustion of ammonium dichromate, and then to use the resulting $\mathrm{RDX} @ \mathrm{Cr}_{2} \mathrm{O}_{3}$ material as gas generating oxidizer in aluminothermic compositions to improve their reactivity [22]. In the most classical sense, nanothermites are energetic materials made up of a metallic oxide such as $\mathrm{Fe}_{2} \mathrm{O}_{3}, \mathrm{MnO}_{2}, \mathrm{WO}_{3}, \mathrm{MoO}_{3}, \mathrm{CuO}$, and $\mathrm{Bi}_{2} \mathrm{O}_{3}$ in the form of submicron-sized powder and of an aluminum nanopowder with typical particle size distribution ranging from 50 to $120 \mathrm{~nm}$ [23]. The thermochemical properties of the most representative thermite compositions were reported by Fischer and Grubelich [24]. In an extended meaning, nanothermites are any combustible compositions containing a significant amount of metallic species, used as fuels or/and oxidizers, in form of fine powders. In the field of nanothermites, oxygenated metallic salts are more and more used as oxidizers instead of metallic oxides to improve reactivity [25-29].

The nanothermite part of NSTEX is prepared by dispersing its components in a liquid phase (e.g., acetonitrile) by ultrasonic agitation. The mixture is recovered by evaporating the liquid under reduced pressure. Nanothermites should be handled with precaution, owing to their particularly high sensitivity, especially to electrostatic discharge (ESD).

The formulation of a NSTEX is achieved by the dry mixing of the nanothermite with the fine explosive powder. This delicate operation is carried out by a vortex mixing and a manual crushing of aggregates with a spatula, which are repeated till obtaining a material with a good homogeneity. No liquid should be used for mixing the components of NSTEX, in order to avoid the dissolution and the growth of the explosive particles.

The main NSTEX feature is their ability to detonate by a transition from deflagration to detonation (TDD). This 
Fig. 1 Macroscopic aspect of some explosive nanopowders prepared by SFE: nano-PETN, nano-RDX and nano-HMX/CL20 from left to right

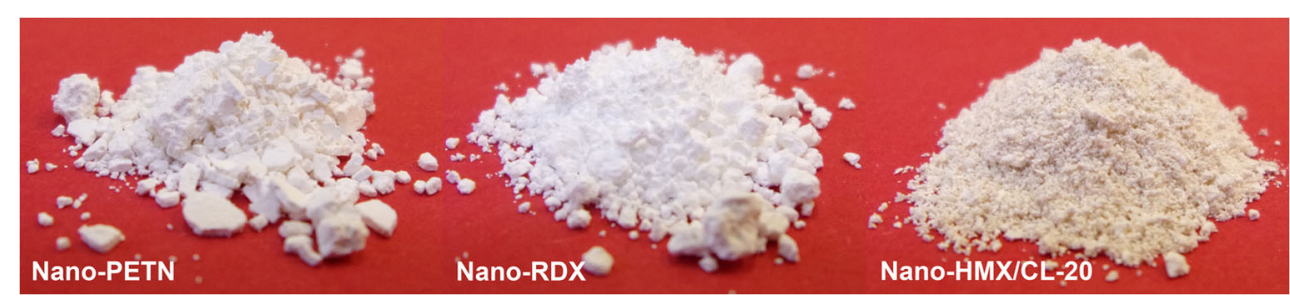

phenomenon is only observed in loose or slightly pressed NSTEX powders. The high power delivered by the fast combustion of the nanothermite activates the detonation of the fine explosive powder, which occurs in short distances (10$20 \mathrm{~mm}$ ). The detonation wave then propagates in the NSTEX charge: Its propagation velocity depends both on the density of the explosive in the composition and on the resistance opposed by the nanothermite particles. In other words, the velocity can be adjusted through the ratio nanothermite/explosive and the density of the NSTEX. The detonation wave produced by the reaction of a NSTEX (e.g., nano-Al/nano- $\mathrm{WO}_{3} /$ nano-RDX:12.4/27.6/60.0 wt.\%) can be transmitted to a PETN secondary charge. This shock to detonation transition is only observed when the detonation velocity of the NSTEX is higher than $3 \mathrm{~km} / \mathrm{s}$ [4]. Figure 2 illustrates the functioning of an experimental detonating device, in a transparent tube, observed with a high speed camera operating at $840000 \mathrm{fr} / \mathrm{s}$. The system comprises of a layer of ignition nanothermite $(5 \mathrm{~mm})$, a NSTEX in loose powder state $(20 \mathrm{~mm})$, a compacted layer of the same NSTEX $(5 \mathrm{~mm})$ and finally a $1 \mathrm{~g}$ PETN charge $(20 \mathrm{~mm})$.

The detonation characteristics of NSTEX make them suitable for the replacement of primary explosives which are salts of heavy metals such as lead or cobalt. Lead azide $\left(\mathrm{PbN}_{6}\right)$ is

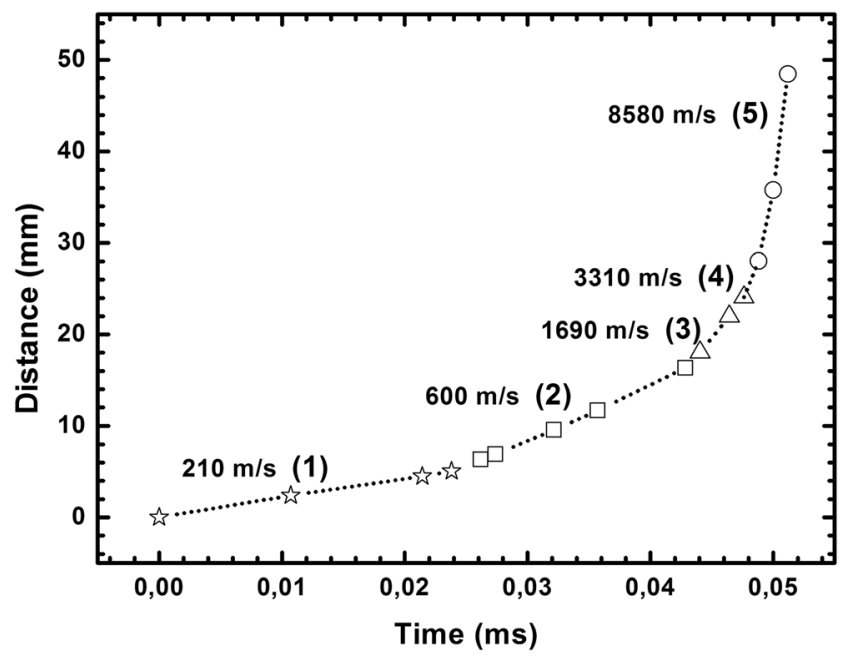

Fig. 2 Curve representing the distance traveled by the flame front according to time, in an experimental device (length $=50 \mathrm{~mm}$ ): burning of the nano- $\mathrm{Al} /$ nano- $\mathrm{CuO}$ nanothermite used for ignition (1), deflagration of a NSTEX composition (2), detonation of the NSTEX (3), acceleration of the detonation wave in a denser NSTEX layer (4), transmission and detonation of a PETN charge (5) the most used primary explosive in detonators: It is synthesized by the Curtius' method from soluble lead salts. On the other hand, the detonation of lead azide produces small lead particles which are dispersed in environment. The persistence of the pollution by lead and its devastating effects on human health, has led European Chemical Agency to put a series of lead compounds on the list of the Substances of Very High Concern (REACH regulation). It was therefore necessary to develop "greener" compositions for replacing traditional primary explosives. From this standpoint, NSTEX are particularly promising, as they can be prepared from benign compounds such as potassium or calcium sulfates [30], aluminum and pentaerythritol tetranitrate.

\section{Fabrication of Macroscopic Materials from Nanoscopic Powders}

The extremely fast flame propagation velocities (FPV) is only observed in nanothermites, in the state of loose powders. The pressing of nanothermites decreases their internal porosity, which considerably slows down the FPV. This effect was observed on $\mathrm{Al} / \mathrm{WO}_{3}$ nanothermites by Prentice et al. [31] and on $\mathrm{Al} / \mathrm{CuO}$ nanothermites by Apperson et al. [32] It is attributed to the fact that the convection of hot gases is made more difficult in denser materials. Moreover, the compression of aluminothermic compositions could slow down the fast spreading of the combustion by the melt dispersion mechanism (MDM) of aluminum [33, 34]. On the other hand, the mixtures of nanopowders of different natures are metastable from a physical point of view, because the phases tend to separate along time by the effect of gravity. The settling changes the local stoichiometry, which alters the pyrotechnics properties of the composition.

Having a high porosity is essential to keep a fast FPV in nanothermites and the most prominent challenge in the forthcoming years will be to develop efficient processes for shaping nanothermite powders into solid, porous objects with good mechanical properties. Various approaches have been explored for this purpose: Tillotson et al. synthesized nanothermites as aerogel monoliths [9], Yan et al. used the electrospinning technique with an energetic polymer as binder to prepare nanothermite mats [35] and Yang et al. simply used filtration to shape nanothermites in membranes [36]. One of the most promising ways was reported by Comet et al. who 
transformed an aluminothermic composition into solid, highly porous objects, by a chemical foaming process $[5,6]$.

The principle of the foaming process is based on the reaction of aluminum nanopowder which is introduced in excess in an aluminothermic mixture $\left(\mathrm{Al} / \mathrm{WO}_{3}\right)$ with an aqueous solution of orthophosphoric acid $\left(\mathrm{H}_{3} \mathrm{PO}_{4}\right)$. The reaction occurs in two exothermic stages: The acid solution first reacts with the oxide shell of aluminum nanoparticles; then, it oxidizes the aluminum core of particles. Water vapor and hydrogen are respectively released by these reactions: In escaping through the reaction medium, these gases create the final porosity of the foam. On the other hand, aluminum phosphate $\left(\mathrm{AlPO}_{4}\right)$ which is produced by the reaction acts as a strong binder (cement) towards the nanoparticles from which the nanothermite is formed. It is worth noting that phosphates and sulfates, which are considered as inert compounds from a pyrotechnic point of view, behave as oxidizers at high temperatures, especially when they are mixed with aluminum nanopowders $[5,30]$. Special caution is required to handle the pastes prepared from concentrated inorganic acids such as $\mathrm{H}_{3} \mathrm{PO}_{4}$ and $\mathrm{H}_{2} \mathrm{SO}_{4}$ and fine aluminum powders, due the high risk of hydrogen explosion [37]. The induction time of the foaming reaction and the maximal temperature of the medium can be controlled through the concentration of the aqueous orthophosphoric acid solution (Fig. 3).

Nanothermites foams have the appearance of concrete pieces; they are quite insensitive to impact, friction and electrostatic discharge (ESD). They can be sawn or drilled without activating their combustion. Once ignited, nanothermite foams combust violently, with a blinding fireball and the release of abundant fumes. The reaction mechanism consists, first in the reduction of aluminum phosphate in phosphorus, which in turns burns in contact with atmospheric oxygen.

Nanothermites in the state of loose powders can be stabilized in thin layers deposited on the surface of the grains of a propulsive powder. For instance, Berthe et al. have coated

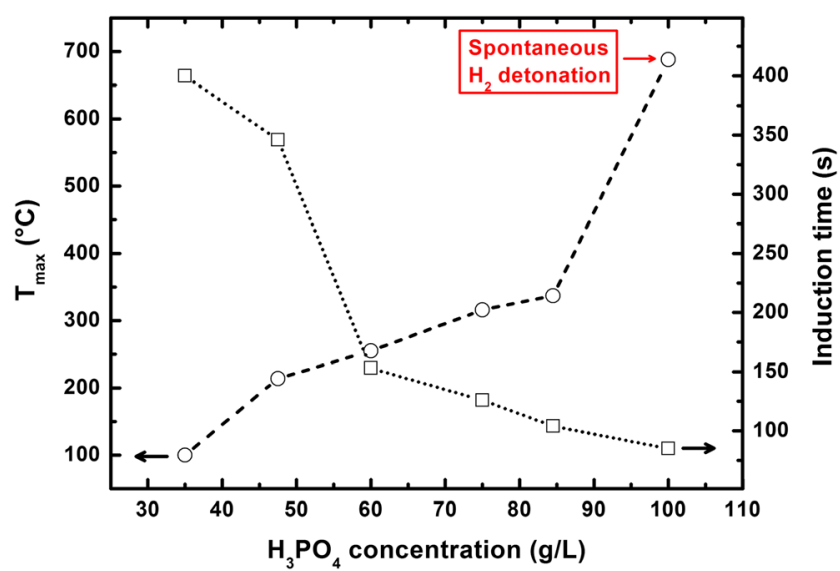

Fig. 3 Curves representing the induction time of the foaming reaction (squares) and the maximum temperature reached during the reaction (circles) depending on the orthophosphoric acid concentration
Vectan A1 powder flakes by a layer of a sulfate-based nanothermite $\left(\mathrm{Al} / \mathrm{Na}_{2} \mathrm{SO}_{4}\right)$ with a thickness of $50 \mu \mathrm{m}$. The addition of only some percents $(10 \mathrm{wt} \%)$ of nanothermite totally changed the ignition and the combustion regime of the propulsive powder. The effect observed was tuned by mixing coated and uncoated Vectan A1 grains in different mass ratios [7]. In these energetic systems, the density of nanothermite layer and the thermal conductivity of the substrate on which it is coated are crucial parameters. Sullivan et al. have deposited $\mathrm{Al} / \mathrm{CuO}$ nanothermite dense layers by electrophoresis on platinum electrodes in miniaturized systems. However, the propagation velocity is smaller than $50 \mathrm{~m} / \mathrm{s}$ owing too high density of the nanothermite deposits $(\approx 29 \%$ of the TMD) [38].

\section{Conclusions}

The use of nanomaterials in the science of pyrotechnics and explosives will lead to important breakthroughs in this domain. Future energetic materials will be greener and safer [39]. Moreover, they will have intrinsic high performance, owing to the fact that they will be designed in such a way to perfectly match with precise needs. Although this trendy research area has its roots in past, black powder and dynamites are indeed historical examples of energetic nanomaterials, much academic and applicative research remains to be done to integrate nanomaterials in operational systems.

The first step was to have the components of energetic systems in the state of nanopowders. The research carried out over the past two decades has led to the commercialization of fine and stable aluminum nanopowders, which are a major component of most of energetic nanomaterials. On the other hand, the Spray Flash Evaporation (SFE) process developed in our laboratory has been used for preparing numerous high explosives in the state of fine or ultrafine nanopowders in quantities which are still compatible with industrial applications.

The fine explosive powders prepared by the SFE process, were mixed with nanothermites to prepare a new family of detonating substances, which were called Nanostructured Thermites and Explosives (NSTEX). The detonation velocity of NSTEX can be set at a desired value, by playing on their composition and on their porosity. NSTEX have similar properties as those of primary explosives, which are molecules comprising toxic metals, such as lead or cobalt. Furthermore, NSTEX can be prepared from benign compounds from a toxicological standpoint, which makes them promising candidates for replacing classical primary explosives.

The last challenge to overcome for the integration of nanomaterials in pyrotechnic systems is the transformation of loose nanopowders, which are metastable in nature, into 
porous, solid and stable objects. Much research remains to be done in this domain, but recent scientific literature reports very promising results for future defense applications $[23,39]$.

Acknowledgments Authors are thankful to the Greener and Safer Energetic and Ballistic Systems committee for having given the opportunity of presenting our work in the 3rd GSEBS conference held in Brest.

Open Access This article is distributed under the terms of the Creative Commons Attribution 4.0 International License (http:// creativecommons.org/licenses/by/4.0/), which permits unrestricted use, distribution, and reproduction in any medium, provided you give appropriate credit to the original author(s) and the source, provide a link to the Creative Commons license, and indicate if changes were made.

Publisher's Note Springer Nature remains neutral with regard to jurisdictional claims in published maps and institutional affiliations.

\section{References}

1. Nobel A (1868) Improved explosive compound, US Letters Patent $78: 317$

2. Risse B, Spitzer D, Hassler D, Schnell F, Comet M, Pichot V, Muhr $\mathrm{H}$ (2012) Continuous formation of submicron energetic particles by the flash-evaporation technique. Chem Eng J 203:158-165. https:// doi.org/10.1016/j.cej.2012.07.032

3. Risse B, Hassler D, Spitzer D (2013) Préparation de nanoparticules par évaporation flash WO2013117671 A1

4. Comet M, Martin C, Klaumünzer M, Schnell F, Spitzer D (2015) Energetic nanocomposites for detonation initiation in high explosives without primary explosives. Appl Phys Lett 107:243108. https://doi.org/10.1063/1.4938139

5. Comet M, Martin C, Schnell F, Spitzer D (2017) Nanothermite foams: from nanopowder to object. Chem Eng J 316:807-812. https://doi.org/10.1016/j.cej.2017.02.009

6. Martin C, Comet M, Schnell F, Spitzer D (2017) Nanothermite with meringue-like morphology: from loose powder to ultra-porous objects. J Vis Exp. https://doi.org/10.3791/56479

7. Berthe J-E, Comet M, Schnell F, Suma Y, Spitzer D (2016) Propellants reactivity enhancement with nanothermites. Propellants Explos Pyrotech 41:994-998. https://doi.org/10.1002/ prep.201600029

8. Gash AE, Satcher JH, Simpson RL, Clapsaddle BJ (2003) Nanostructured energetic materials with sol-gel methods. MRS Online Proc Libr Arch 800

9. Tillotson TM, Gash AE, Simpson RL, Hrubesh LW, Satcher JH Jr, Poco JF (2001) Nanostructured energetic materials using sol-gel methodologies. J Non-Cryst Solids 285:338-345

10. Kumar R, Siril PF, Soni P (2015) Optimized synthesis of HMX nanoparticles using antisolvent precipitation method. J Energ Mater 33:277-287. https://doi.org/10.1080/07370652.2014. 988774

11. Lee B-M, Jeong J-S, Lee Y-H, Lee B-C, Kim H-S, Kim H, Lee Y-W (2009) Supercritical antisolvent micronization of cyclotrimethylenetrinitramin: influence of the organic solvent. Ind Eng Chem Res 48:11162-11167. https://doi.org/10.1021/ ie 900448 w

12. Anandharamakrishnan C, Ishwarya SP (2015) Spray drying techniques for food ingredient encapsulation. John Wiley \& Sons, Ltd, The Atrium, Southern Gate, Chichester, West Sussex, UK ; Hoboken, NJ
13. Qiu H, Stepanov V, Di Stasio AR, Surapaneni A, Lee WY (2015) Investigation of the crystallization of RDX during spray drying. Powder Technol 274:333-337. https://doi.org/10.1016/j.powtec. 2015.01.032

14. Stepanov V, Krasnoperov LN, Elkina IB, Zhang X (2005) Production of nanocrystalline RDX by rapid expansion of supercritical solutions. Propellants Explos Pyrotech 30:178-183. https:// doi.org/10.1002/prep.200500002

15. Essel JT, Cortopassi AC, Kuo KK, Leh CG, Adair JH (2012) Formation and characterization of nano-sized RDX particles produced using the RESS-AS process. Propellants Explos Pyrotech 37: 699-706. https://doi.org/10.1002/prep.201100139

16. Klaumünzer M, Hübner J, Spitzer D (2016) Production of energetic nanomaterials by Spray Flash Evaporation. World Acad Sci Eng Technol 10:1191-1195

17. Séve A, Pichot V, Schnell F, Spitzer D (2017) Trinitrotoluene nanostructuring by Spray Flash Evaporation process. Propellants Explos Pyrotech 42:1051-1056. https://doi.org/10.1002/prep. 201700024

18. Risse B, Schnell F, Spitzer D (2014) Synthesis and desensitization of nano- $\beta$-HMX. Propellants Explos Pyrotech 39:397-401. https:// doi.org/10.1002/prep.201300161

19. Deckert-Gaudig T, Pichot V, Spitzer D, Deckert V (2017) Highresolution Raman spectroscopy for the nanostructural characterization of explosive nanodiamond precursors. ChemPhysChem 18: 175-178. https://doi.org/10.1002/cphc.201601276

20. Spitzer D, Risse B, Schnell F, Pichot V, Klaumünzer M, Schaefer MR (2014) Continuous engineering of nano-cocrystals for medical and energetic applications. Sci Rep 4. https://doi.org/10.1038/ srep06575

21. Le Brize A, Spitzer D (2016) Plasticization of submicron-structured LOVA propellants by a linear dinitramine. Cent Eur J Energ Mater 13:547-556

22. Comet M, Siegert B, Pichot V, Gibot P, Spitzer D (2008) Preparation of explosive nanoparticles in a porous chromium(III) oxide matrix: a first attempt to control the reactivity of explosives. Nanotechnology 19:285716. https://doi.org/10.1088/0957-4484/ $19 / 28 / 285716$

23. Lafontaine E, Comet M (2016) Nano-thermites. ISTE Ltd/John Wiley and Sons Inc, Hoboken, NJ

24. Fischer SH, Grubelich MC (1996) A survey of combustible metals, thermites, and intermetallics for pyrotechnic applications. Sandia National Laboratories

25. Wang S, Liu X, Schoenitz M, Dreizin EL (2017) Nanocomposite thermites with calcium iodate oxidizer. Propellants Explos Pyrotech 42:284-292. https://doi.org/10.1002/prep.201600213

26. Zhou W, DeLisio JB, Wang X, Zachariah MR (2017) Reaction mechanisms of potassium oxysalts based energetic composites. Combust Flame 177:1-9. https://doi.org/10.1016/j.combustflame. 2016.05.024

27. Brusnahan JS, Shaw AP, Moretti JD, Eck WS (2017) Periodates as potential replacements for perchlorates in pyrotechnic compositions. Propellants Explos Pyrotech 42:62-70. https://doi.org/10. 1002/prep.201600084

28. Wang H, Jian G, Zhou W, DeLisio JB, Lee VT, Zachariah MR (2015) Metal iodate-based energetic composites and their combustion and biocidal performance. ACS Appl Mater Interfaces 7: 17363-17370. https://doi.org/10.1021/acsami.5b04589

29. Wu T, Wang X, Zavalij PY, DeLisio JB, Wang H, Zachariah MR (2018) Performance of iodine oxides/iodic acids as oxidizers in thermite systems. Combust Flame 191:335-342. https://doi.org/ 10.1016/j.combustflame.2018.01.017

30. Comet M, Vidick G, Schnell F, Suma Y, Baps B, Spitzer D (2015) Sulfates-based nanothermites: an expanding horizon for metastable interstitial composites. Angew Chem Int Ed 54:4458-4462. https:// doi.org/10.1002/anie.201410634 
31. Prentice D, Pantoya ML, Gash AE (2006) Combustion wave speeds of sol-gel-synthesized tungsten trioxide and nano-aluminum: the effect of impurities on flame propagation. Energy Fuel 20:23702376. https://doi.org/10.1021/ef060210i

32. Apperson S, Shende RV, Subramanian S, Tappmeyer D, Gangopadhyay S, Chen Z, Gangopadhyay K, Redner P, Nicholich S, Kapoor D (2007) Generation of fast propagating combustion and shock waves with copper oxide/aluminum nanothermite composites. Appl Phys Lett 91:243109. https://doi. org/10.1063/1.2787972

33. Levitas VI, Asay BW, Son SF, Pantoya M (2007) Mechanochemical mechanism for fast reaction of metastable intermolecular composites based on dispersion of liquid metal. J Appl Phys 101:083524. https://doi.org/10.1063/1.2720182

34. Levitas VI, Asay BW, Son SF, Pantoya M (2006) Melt dispersion mechanism for fast reaction of nanothermites. Appl Phys Lett 89: 071909. https://doi.org/10.1063/1.2335362
35. Yan S, Jian G, Zachariah MR (2012) Electrospun nanofiber-based thermite textiles and their reactive properties. ACS Appl Mater Interfaces 4:6432-6435. https://doi.org/10.1021/am3021125

36. Yang Y, Zhang Z-C, Wang P-P, Zhang J-C, Nosheen F, Zhuang J, Wang X (2013) Hierarchical $\mathrm{MnO}_{2} / \mathrm{SnO}_{2}$ heterostructures for a novel free-standing ternary thermite membrane. Inorg Chem 52: 9449-9455. https://doi.org/10.1021/ic401068n

37. Martin C, Comet M, Schnell F, Berthe J-E, Spitzer D (2018) Aluminum nanopowder: a substance to be handled with care. J Hazard Mater 342:347-352. https://doi.org/10.1016/j.jhazmat.2017. 08.018

38. Sullivan KT, Kuntz JD, Gash AE (2012) Electrophoretic deposition and mechanistic studies of nano- $\mathrm{Al} / \mathrm{CuO}$ thermites. J Appl Phys 112:024316. https://doi.org/10.1063/1.4737464

39. Comet M, Martin C, Schnell F, Spitzer D (2019) Nanothermites: a short review. Factsheet for experimenters, present and future challenges. Propellants Explos Pyrotech 44:18-36. doi: https://doi.org/ 10.1002/prep.201800095 